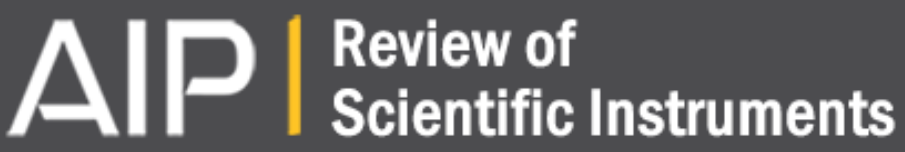

Electric measurements with constant current: A practical method for characterizing dielectric films

José A. Giacometti, Célio Wisniewski, Paulo Antonio Ribeiro, and Walterley Araújo Moura

Citation: Review of Scientific Instruments 72, 4223 (2001); doi: 10.1063/1.1409564

View online: http://dx.doi.org/10.1063/1.1409564

View Table of Contents: http://scitation.aip.org/content/aip/journal/rsi/72/11?ver=pdfcov

Published by the AIP Publishing

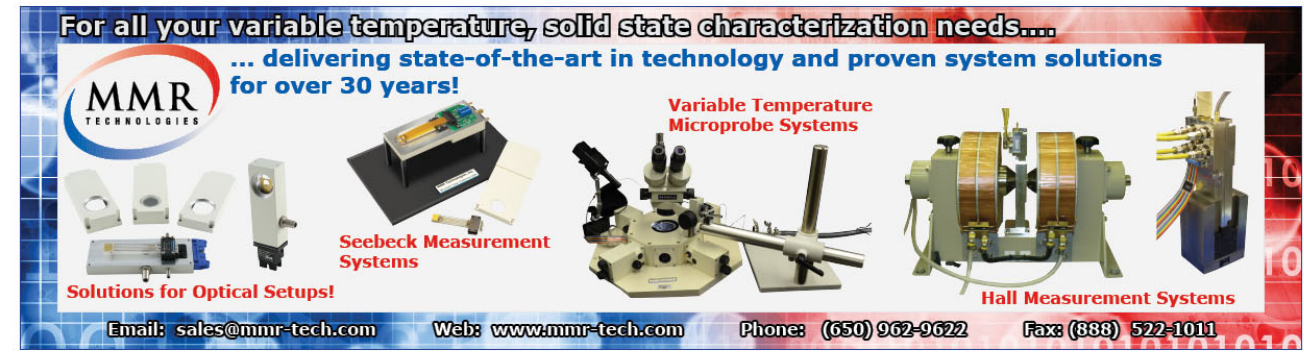




\title{
Electric measurements with constant current: A practical method for characterizing dielectric films
}

\author{
José A. Giacomettia) \\ Faculdade de Ciência e Tecnologia, Universidade Estadual Paulista, 10060-900, Presidente Prudente, SP, \\ Brazil \\ Célio Wisniewski \\ Instituto de Física de São Carlos, Universidade de São Paulo, CP 369. 13566-970, São Carlos, SP, Brazil \\ Paulo Antonio Ribeiro \\ CeFITec, Departamento de Física, Faculdade de Ciências e Tecnologia, Universidade Nova de Lisboa. \\ 2825-114 Caparica, Portugal \\ Walterley Araújo Moura \\ Escola Técnica Federal de Mato Grosso. 78005-390, Cuiabá, MT, Brazil
}

(Received 13 February 2001; accepted for publication 26 June 2001)

\begin{abstract}
This article assesses the use of the constant current (CC) method for characterizing dielectric films. The method is based on charging the sample with a constant current (current stress) and measuring the corresponding voltage rise under the closed circuit condition. Our article shows that the CC method is an alternative to the constant voltage stressing method to study the electric properties of nonpolar, ferroelectric, and polar polymers. The method was tested by determining the dielectric constant of polytetrafluoroethylene, and investigating the electric conduction in poly(ethylene terephthalate). For the ferroelectric polymer poly(vinylidene fluoride), it is shown that hysteresis loops and the dependence of the ferroelectric polarization on the electric field can be obtained. (C) 2001 American Institute of Physics. [DOI: 10.1063/1.1409564]
\end{abstract}

\section{INTRODUCTION}

Electric measurements can be performed by either applying a voltage (voltage stress) to the sample and measuring the electric current, or by charging the sample with a constant current (CC) (current stress) and measuring the voltage rise. CC sources have been available for many years in voltage/current source supplies. ${ }^{1}$ Current stress has a wide application in electrochemistry, ${ }^{2}$ breakdown measurements in semiconductors, ${ }^{3}$ scanning tunneling microscopy, ${ }^{4}$ and measurements of surface electric conductivity using the fourpoint method. ${ }^{5}$ The CC approach has also been used in connection with corona charging of insulators. In the 1980's, a corona triode was built which allowed dielectric samples to be charged with a CC, under an open circuit. ${ }^{6-8}$ In addition to charging control, this corona triode permitted the investigation of electrical properties of polymers ${ }^{9,10}$ and even to obtain hysteresis curves for ferroelectric polymers. ${ }^{11}$ In this work, the CC method is applied in measurements under the closed circuit condition, i.e., with electroded samples.

The mathematical treatment of experimental data is not the same in voltage and current stresses. In controlled current experiments, equations are based on the known current, while in voltage controlled methods, they are based on the known voltage. The advantage of the CC is that the Maxwell total current equation can be, in some cases, analytically integrated and the charge transport and trapping in insulators may be theoretically treated. ${ }^{9,12}$ The connection between the

${ }^{\text {a)} E l e c t r o n i c ~ m a i l: ~ w w w . p o l i m e r o s . i f . s c . u s p . b r ~ a n d ~ g i a c o m e t t i @ i f . s c . u s p . b r ~}$
$\mathrm{CC}$ and voltage methods is briefly discussed in Ref. 13. More recently, the equivalence between hysteresis loops obtained with the CC and the Saywer-Tower methods was already demonstrated.$^{14}$ From the experimental point of view, another advantage of the $\mathrm{CC}$ method is that an ammeter is not required.

This article assesses the use of the $\mathrm{CC}$ method for characterizing dielectric films such as polymers. The $\mathrm{CC}$ controller circuit, designed to be used in combination with a commercial high-voltage (HV) bipolar amplifier, is described. The setup is fully computerized allowing an electric current as low as a few nA to be monitored. The basic equations of the CC method are presented, which allows interpretation of the various types of measurements in dieletric films. Here, in particular, results will be discussed for the measurement of the dielectric constant in nonpolar/polar polymer samples and for hysteresis loops in ferroelectric polymers.

The article is organized as follows. In the Experiment section, Sec. II, samples, experimental conditions, the CC controller circuit, and its characteristics are described. The basic equations of the CC method are given in Sec. III and in Secs. IV-VI, results are presented for different polymers. Final remarks are highlighted in Sec. VII.

\section{EXPERIMENT}

\section{A. The constant current setup and the $\mathrm{CC}$ controller characteristics}

The CC method consists in charging the sample with a constant electric current, $I_{0}$, and measuring the resulting 


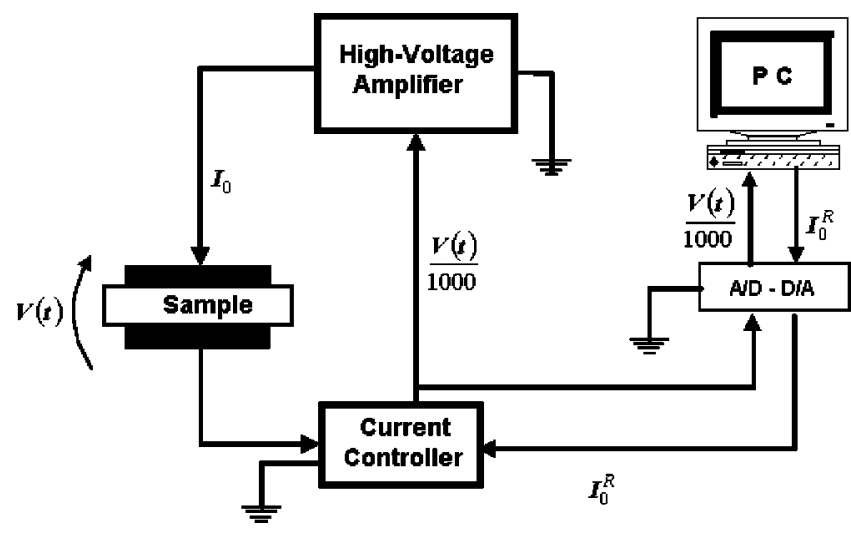

FIG. 1. Schematic diagram of the measuring setup. $V(t)$ is the measured voltage and $I_{0}$ is the constant current.

voltage rise, $V(t)$, on its electrodes. The schematic diagram of the measuring setup is illustrated in Fig. 1. It consists of a homemade constant current controller, a HV bipolar amplifier TREK 610C (maximum voltage of $\pm 10 \mathrm{kV}$ ), the dielectric sample, and a personal computer with digital-to-analog (D/A) and analog-to-digital (A/D) converters from National Instruments (12 bits of resolution). The controller circuit, shown in Fig. 2, is the most critical part of the setup. It includes a current amplifier/current-to-voltage converter, CA, an error amplifier, $E$, and an integrator, $I$, that operates in the proportional-integrative mode (PI). A follower amplifier, $F$, was also added to the circuit allowing the current to be monitored during the experiments. $R_{1}$ and $C_{1}$ allow adjusting the current sensitivity and the variable resistor, $R_{X}$, allows adjusting the integrator time constant. The error amplifier $E$, with a unitary gain, gives the signal difference between the reference voltage $V_{R}$, and the measured signal, $V_{0}$. The amplifier CA converts the current $I_{0}$ into the voltage $V_{0}$. If $V_{0}-V_{R}>0$, the integrator $I$ decreases its output, then decreasing $V(t)$ and $I_{0}$. If $V_{0}-V_{R}<0$, then $V(t)$ and $I_{0}$ are increased, forcing $V_{0}=V_{R}$ and, thus, the current in the circuit to be constant.

The computer software, written in Visual Basic for Win-

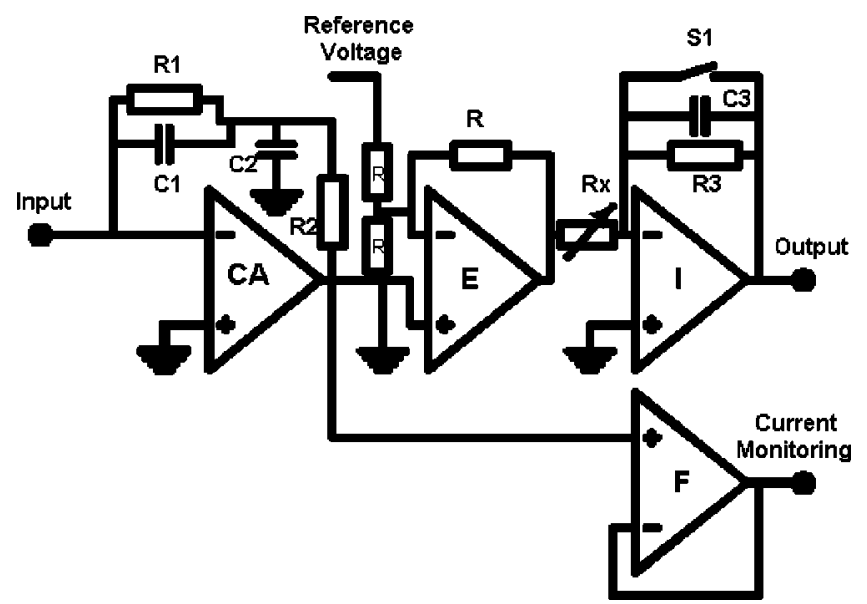

FIG. 2. Circuit diagram of the current controller. CA is the current amplifier, $\mathrm{E}$ an error amplifier, and I the integrator. The circuit component values are $R_{1}=100 \mathrm{M} \Omega, R_{2}=500 \Omega, R_{3}=10 \mathrm{k} \Omega, R=15 \mathrm{k} \Omega, 1 \mathrm{k} \Omega<R_{x}<10 \mathrm{M} \Omega$, $C_{1}=100 \mathrm{pF}, C_{2}=10 \mathrm{nF}$, and $C_{3}=100 \mathrm{nF}$. The integrated circuits are $\mathrm{CA} 1=\mathrm{CA} 3140, E=\mathrm{LF} 356 \mathrm{~N}$ and $I=\mathrm{LF} 356 \mathrm{~N}$. dows, allows setting the value of the reference voltage $V_{R}$ (50 $\mathrm{mV}$ corresponds to $1 \mathrm{nA}$ ) to the CC controller via the D/A computer interface. Via computer, the HV output of the Trek amplifier can be activated remotely, and the switch S may also be actuated, starting and stopping the operation of the CC controller. The experimental data, $V(t)$ and $I_{0}$, are collected via the A/D interface, displayed graphically on real time on the computer screen and stored in the hard disk. With the software, the measurement may be discontinued automatically (opening $S$ and turning the HV supply off), whenever the sample breaks down.

The setup can be operated in the voltage interval of $\pm 10 \mathrm{kV}$ and one of its capabilities is to reverse the polarity of the current without discontinuing the measurement. Control experiments demonstrated that the switching time from one polarity to another is less than a second, much smaller than the several minutes of a typical measurement. The controller can be operated with a current of a few nA and above, within a few percent of fluctuation.

\section{B. Experimental conditions and samples}

Samples of polytetrafluoroethylene, Teflon polytetrafluoroethylene (PTFE), were supplied by Dilectricx, USA. Poly(vinylidene fluoride), PVDF, samples were biaxially stretched and supplied by Kureha, Japan, and samples of poly(ethylene terephthalate), PET, were supplied by Rhodia, Brazil. The sample thickness used in this work was $12 \mu \mathrm{m}$. The sample electrodes of PVDF and Teflon PTFE, were deposited by thermal evaporation of aluminum in a vacuum, had diameter of $1 \mathrm{~cm}$ corresponding to an area of $0.8 \mathrm{~cm}^{2}$. In the case of PET samples, the electrodes had $2 \mathrm{~cm}$ of diameter corresponding to the area of $3.1 \mathrm{~cm}^{2}$. The sample holder allows one to perform measurements in a high vacuum and the temperature can be controlled within $\pm 0.5^{\circ} \mathrm{C}$.

\section{CC BASIC EQUATIONS}

The Maxwell total current density $J(t)$ in a dielectric slab in planar geometry is given by

$$
J(t)=J_{C}(t)+\frac{d D(t)}{d t},
$$

where $\quad J_{C}(t)=(1 / L) \int_{0}^{L} J_{C}(x, t) d x \quad$ and $\quad D(t)$ $=(1 / L) \int_{0}^{L} D(x, t) d x$ are mean values of the conduction current and electric displacement ( $x$ is the coordinate and $L$ the sample thickness). The electric displacement is given by

$$
D(t)=\epsilon E(t)+P(t),
$$

where $\epsilon$ is the instantaneous dielectric permittivity, and $E$ and $P$ are the electric field and the electric polarization, respectively. In general, the electric polarization could be time delayed or ferroelectric, which has a nonlinear dependence on the electric field.

For the constant current case, $J(t)=J_{0}$, Eq. (1) becomes

$$
J_{0}=\epsilon \frac{d E(t)}{d t}+\frac{d P(t)}{d t}+J_{C}(t)
$$




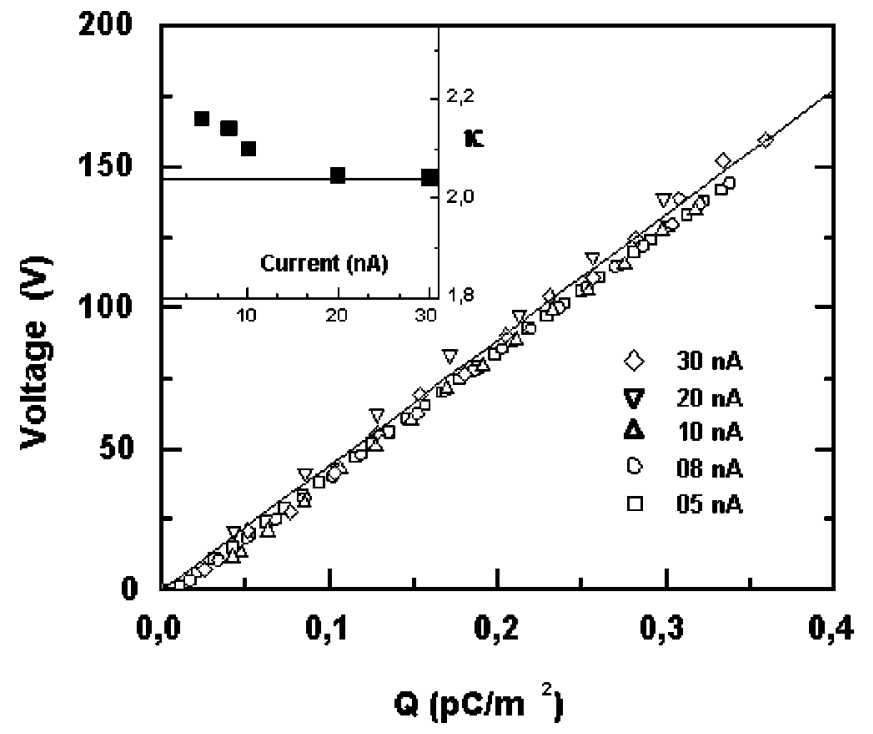

FIG. 3. Voltage buildup plotted versus the charge density, $Q(t)=J_{0} t$, transferred to the Teflon PTFE samples charged with different values of current. Inset: the dielectric constant determined from the voltage build rate for different charging currents. Sample thickness is $12 \mu \mathrm{m}$.

$$
I_{0}=C \frac{d V(t)}{d t}+A \frac{d P(t)}{d t}+I_{C}(t)
$$

with the voltage on the sample given by $V(t)=E(t) L$, sample capacitance $C=\epsilon A / L$ and $I_{C}(t)=A J_{C}(t), I_{0}=A J_{o}$, and $A$ is the sample area. It is worth mentioning that Eq. (4) can be analytically integrated, in some cases, if $P(t)$ and $I_{C}(t)$ are known. For further details see Refs. 9 and 12.

When samples are nonpolar $[P(t)=0]$ and there is no electric conduction $\left(I_{C}(t)=0\right)$, Eq. (4) becomes

$$
V(t)=L \frac{J_{0} t}{\epsilon}=\frac{I_{0} t}{C},
$$

leading to a linear voltage increase with a rate equal to $I_{0} / C$. One can infer from Eqs. (4) and (5) that the rate $I_{0} / C$ will be the upper limit since the presence of polarization or conduction will produce a decrease on the rate of the voltage increase.

\section{PERFORMANCE OF THE CC METHOD}

For evaluating the performance of the $\mathrm{CC}$ method for polymers such as Teflon PTFE, the voltage buildup was measured and the dielectric constant determined. Because Teflon PTFE is a nonpolar polymer, $d P(t) / d t=0$ in Eq. (4), thus

$$
I_{0}=C \frac{d V(t)}{d t}+I_{C}(t)
$$

Figure 3 shows experimental points corresponding to the voltage increase for $12 \mu \mathrm{m}$ thick Teflon PTFE samples, plotted as a function of the charge density $Q(t)=J_{0} t$, for different CC current amplitudes. It is well known that Teflon is a very highly insulating material in which the electric conduction could be neglected for moderate electric fields, thus, Eq. (6) is reduced to Eq. (5). In fact, the experimental data $V(t) \times Q(t)$ in Fig. 3 show that the voltage increases linearly up to $200 \mathrm{~V}$, corresponding to an electric field of $17 \mathrm{MV} / \mathrm{m}$.

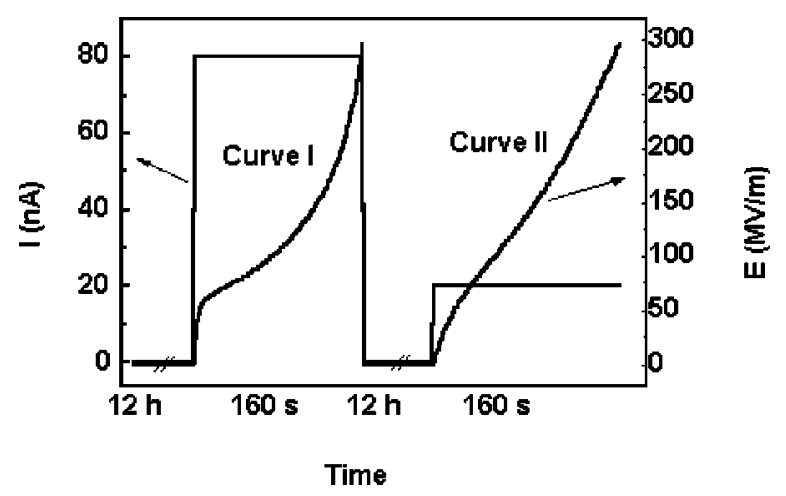

FIG. 4. Potential buildups for a PVDF ferroelectric sample. Curve I corresponds to the voltage increase during the switching of the polarization. Curve II sample charged with the same voltage polarity without polarization switching. Sample thickness is $12 \mu \mathrm{m}$.

From the slopes, the relative dielectric constant was calculated and the results are plotted in the inset of Fig. 3 as a function of $I_{0}$. The deviation from $\kappa=2.05$ increases for small amplitudes of $I_{0}$, since the conduction current can not be neglected when compared with the displacement current [see Eq. (1)]. For comparison, the full line in Fig. 3 was plotted using Eq. (5). Calculation assumed that the sample had a relative dielectric constant, $\kappa=\epsilon / \epsilon_{0}=2.05$, which is typical for Teflon PTFE. Similar results for polyfluoroethylene propylene, Teflon FEP, (not shown here) were obtained.

After the initial, linear increase, the voltage reached the steady state (not shown in the Fig. 3), i.e., a saturation value for the PTFE samples. At the steady state $d V(t) / d t=0$, consequently, the electric conduction through the sample is equal to the charging current [cf. Eq. (4)]. CC measurements in the corona charging of FEP samples were described in our previous papers. ${ }^{15-18}$ For example, when samples were negatively, corona charged results were explained using theoretical models assuming a near trapping filling limit. ${ }^{6,17}$

\section{APPLICATION OF THE CC METHOD TO A FERROELECTRIC POLYMER}

Figure 4 shows the voltage buildups in a PVDF sample charged with a positive current density of $8.0 \mathrm{~mA} / \mathrm{m}^{2}\left(I_{0}\right.$ $=80 \mathrm{nA}$ ). Curve I corresponds to the voltage increase when the ferroelectric switching occurs. It increases linearly at the very beginning at a rate equal to $I_{0} / C$, where the electric capacitance, $C$, is equal to $0.75 \mathrm{nF}$ in good agreement with $\kappa=13$, usually found in the literature. ${ }^{19}$ After this initial, linear increase, the curve shows a quasiplateau, and finally increases steeply again. The quasiplateau region is related to the ferroelectric dipole switching which leads to the neutralization of the incoming charge. In Curve II [sample poled with the same current polarity as in Curve I but the current density is $\left.2.5 \mathrm{~mA} / \mathrm{m}^{2}\left(I_{0}=20 \mathrm{nA}\right)\right]$, the potential grows more rapidly since switching does not occur, i.e., in this case, the incoming charge is not neutralized by the switching of the ferroelectric polarization. In our measurements, we took care to reduce the electric conduction in PVDF to a negligible value by performing the measurements in high vacuum, ${ }^{20}$ i.e., $J_{C}(t)=0$ in Eq. (3). 


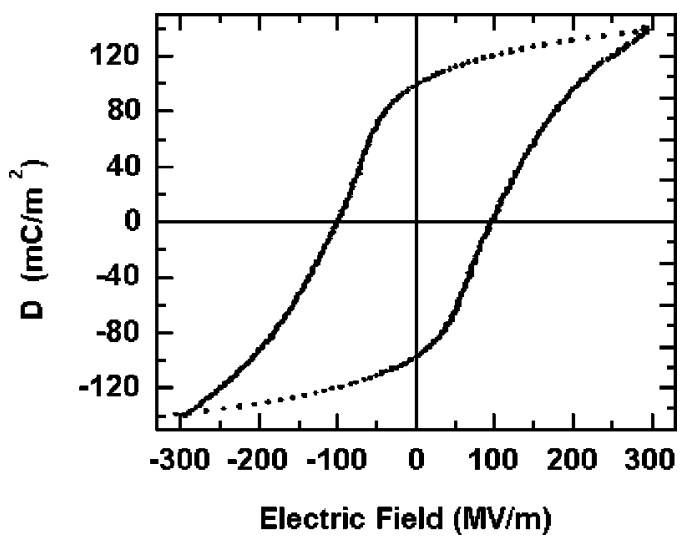

FIG. 5. Hysteresis loop of the electric displacement, D, versus the electric field, E, for the PVDF sample $12 \mu \mathrm{m}$ thick.

The ferroelectric hysteresis loop, with the electric displacement $D$ versus the electric field $E$, can be obtained if the electric conduction in the samples can be neglected. From Eq. (1), with $J_{C}(t)=0$, the electric displacement is $D(t)=I_{0} t$. Since $E(t)=V(t) / L$, by eliminating the time one can obtain the $D \times E$ loops. For obtaining the hysteresis loops, experimental measurements are performed continuously, i.e., after a positive charging, the polarity of the electric current is reversed. Figure 5 displays the resulting hysteresis plot. A slow increase of $D$ versus $E$ in the range of very high fields is seen, which is due to the contribution of the displacement current, $C d V(t) / d t$ [see Eq. (4)]. Thus, in order to obtain loops of electric polarization, $P \times E$, it is necessary to subtract the $\epsilon$ related contribution, i.e., the displacement current is subtracted from the total current. Results with the CC method agreed very well with those obtained from the Sawyer-Tower setup (applying a sinusoidal or a triangle voltage). ${ }^{14}$ It was also shown that the CC method was suitable for determining the dependence of the stable ferroelectric polarization on the electric field in PVDF samples. ${ }^{20}$ To do it, successive charging experiments were performed with the same polarity. Experimental procedures and results are discussed in detail in Ref. 20.

\section{ELECTRIC CONDUCTION IN PET}

Another possible application of the CC method is the study of electric conduction in polymers. As an example, we will present results for PET. Figure 6 shows the buildup of the voltage for PET samples charged with a CC current density of $63.6 \mu \mathrm{A} / \mathrm{m}^{2} \quad\left(I_{0}=20 \mathrm{nA}\right)$ at various temperatures. Measurements showed that after the linear increase $V(t)$ reached the steady state, where the CC current impinging on the sample is equal to the conduction current through the sample. Lower values of the saturation voltage indicate higher conduction.

We investigated the dependence of the steady state voltage on the charging current for different values of temperature. Analysis of data, see Fig. 7, indicated that the conduction current obeys the Poole-Frenkel law, i.e., a linear dependence of the logarithm of the electric conductivity $(J / E)$ versus $\sqrt{E}$. In the Poole-Frenkel model, the slope of $J / E$ versus $\sqrt{E}$ is equal to $\beta_{F} / 2 k T$, where $k$ is the Boltzmann

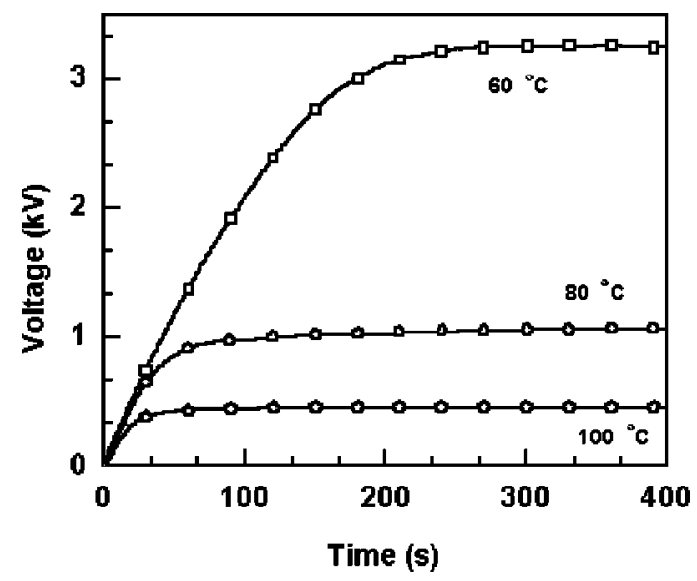

FIG. 6. Voltage buildup for PET samples for different temperatures. The CC electric current was $20 \mathrm{nA}$. Sample thickness is $12 \mu \mathrm{m}$.

constant, $T$ is the absolute temperature, and $\beta_{F}$ $=\left(e^{3} / \pi \epsilon\right)^{1 / 2}$ is the Poole-Frenkel constant $(e$ is the electronic charge). From the plot of Fig. 7, reasonable values for the dielectric constant were obtained, ${ }^{21}$ confirming that the Poole-Frenkel conduction model is appropriate to explain the experimental results with the CC method. This is not surprising since it is well known that the Poole-Frenkel conduction plays an important role in PET polymer. ${ }^{22}$

\section{DISCUSSION}

Measurements with constant current are frequently used to control the corona charging of insulators, usually under the open circuit condition. ${ }^{9}$ According to our knowledge, this work is pioneering because it assesses the electric characterization of different dielectric materials using the CC method. Our experimental setup was designed for measurements using electroded samples, i.e., under the closed circuit condition. Basic equations of the $\mathrm{CC}$ method are the same employed for the $\mathrm{CC}$ open circuit in the corona charging. The application of our CC method to investigate dielectric properties of insulating films was shown. When charged up to moderate electric fields, Teflon PTFE displays a linear increase in voltage, which allows the dielectric constant of the sample to be measured as a test procedure for evaluating the

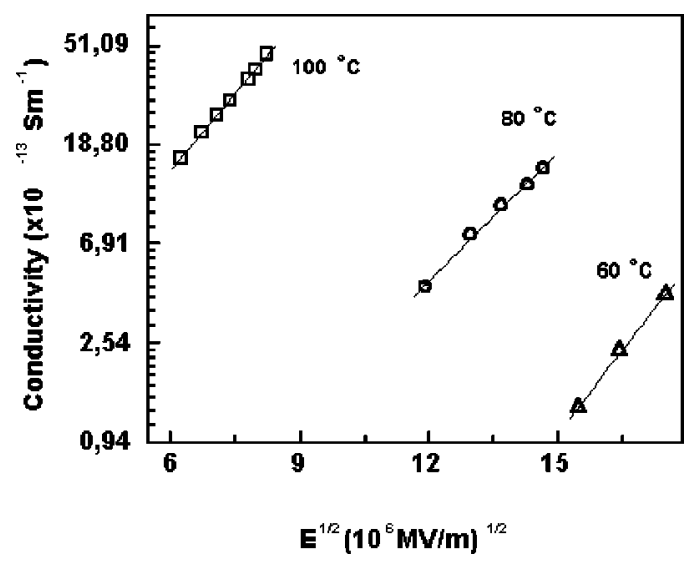

FIG. 7. Dependence of the electric conductivity on the square root of the electric field (at steady state condition) for different temperatures. Sample thickness is $12 \mu \mathrm{m}$. 
CC method. For PVDF, we show that the switching of the ferroelectric polarization can be observed in successive measurements with the same current polarity. We also showed that hysteresis loops of PVDF samples can be obtained if measurements are performed continuously, i.e., the polarity of the current is reversed while the current is continuously measured. ${ }^{14}$ Finally, it was shown that the electric conduction in PET obeys the Poole-Frenkel law in agreement with published data. $^{21,22}$

Theoretical models for CC measurements are already available and described in the literature ${ }^{12}$ for several cases such as free charge injection and charge injection in the presence of trapping. Such models were originally applied to corona charging measurements under open circuit but they are the same for closed circuit measurements. The main difference resides in the fact that in corona measurements, one surface usually has no electrode and corona ions are deposited on it. In contrast, our measurements are performed with samples having two electrodes that could produce, for example, different charge injection processes.

Overall, we stress that the CC method is a convenient method for characterizing dielectrics. Its main advantage is related to the theoretical interpretation of experimental data since the differential equations of the charging transport can be analytically integrated.

\section{ACKNOWLEDGMENTS}

FAPESP, CNPq, and CAPES (Brazil) financially supported this work.
${ }^{1}$ R. W. Haisty, Rev. Sci. Instrum. 31, 1297 (1960).

${ }^{2}$ A. J. Brad and L. R. Faulkner, Electrochemical Methods (Wiley, New York, 1980), Vol. 1, Chap. 7, pp. 249-279.

${ }^{3}$ K. Eriguchi and Y. Uraoka, Jpn. J. Appl. Phys., Part 1 35, 1535 (1996).

${ }^{4}$ G. Binning and H. Rohrer, Rev. Mod. Phys. 71, S324 (1999).

${ }^{5}$ F. M. Smith, Bell Bull. Tech. Journal 20, 711 (1958).

${ }^{6}$ B. Gross, J. A. Giacometti, and G. F. Leal Ferreira, CEIDP Annual Report 87, 0 (1981).

${ }^{7}$ J. A. Giacometti, J. Phys. D 20, 675 (1987).

${ }^{8}$ J. A. Giacometti and J. C. Sinézio Campos, Rev. Sci. Instrum. 61, 1143 (1990).

${ }^{9}$ J. A. Giacometti and O. N. Oliveira Jr., IEEE Trans. Electr. Insul. 27, 924 (1992).

${ }^{10}$ J. A. Giacometti, S. Fedosov, and M. M. Costa, Braz. J. Phys. 29, 269 (1999).

${ }^{11}$ N. Alves, J. A. Giacometti, and O. N. Oliveira, Jr., J. Appl. Phys. 62, 1840 (1991).

${ }^{12}$ G. F. Leal Ferreira and M. T. Figueiredo, IEEE Trans. Dielectr. Electr. Insul. 27, 719 (1992).

${ }^{13}$ P. J. Needham, J. E. Parrot, and P. Williams, J. Phys. D 11, 1351 (1978).

${ }^{14}$ J. A. Giacometti, C. Wisniewski, W. A. Moura, and P. A. Ribeiro, Rev. Sci. Instrum. 70, 2699 (1999).

${ }^{15}$ B. Gross, J. A. Giacometti, and G. F. Leal Ferreira, Appl. Phys. A: Solids Surf. 42, 89 (1985).

${ }^{16}$ J. A. Giacometti, G. F. Leal Ferreira, and B. Gross, Phys. Status Solidi A 88, 297 (1985).

${ }^{17}$ G. F. Leal Ferreira and O. N. Oliveira, Jr., Phys. Status Solidi A 105, 531 (1988).

${ }^{18}$ O. N. Oliveira, Jr. and G. F. Leal Ferreira, Appl. Phys. A: Solids Surf. 42, 213 (1987).

${ }^{19}$ T. Furukawa, Phase Transitions 18, 143 (1989).

${ }^{20}$ C. Wisniewski, G. F. Leal Ferreira, W. A. Moura, and J. A. Giacometti, J. Phys. D 33, 2483 (2000).

${ }^{21}$ E. Neagu, P. Pissis, L. Apekis, and J. L. Gomez Ribelles, J. Phys. D 30, 1551 (1997).

${ }^{22}$ E. R. Neagu and R. M. Neagu, Mater. Lett. 34, 364 (1998). 